\title{
Dos manuscritos italianos de medicina en bibliotecas españolas ${ }^{1}$
}

\author{
María Teresa Navarro Salazar *
}

\begin{abstract}
RESUMEN
ABSTRACT

En la biblioteca Nacional de Madrid y In the National Library in Madrid an in en la Capitular de Toledo se hallan dos manuscritos médicos italianos, inéditos, que desarrollan aspectos diferentes de la medicina, desde el punto de vista argumental $y$ metodológico. Se trata del Spechio di medicina de Aldobrandino di Berto, $y$ del De arthetica passione, del doctor

Antonio Guainiero de Pauia. El Spechio reúne una serie de recetas heterogéneas, mientras el tratado De Arthetica se concentra en el estudio de una única enfermedad, la gota.

Las diferencias conceptuales, metodológicas y lingüísticas existentes entre ambos, constituyen un buen ejemplo para poder analizar cómo se lleva a cabo la transmisión de conocimientos médicos a finales de la the Capitular Library in Toledo there are two Italian medical manuscripts, unpublished, which involve different medical aspects from the points of view of content and method. They are Spechio di medicina by Aldobrandino di Berto and De arthetica passione by Dr. Antonio Guainiero de Pavía. The former contains a number of heterogeneous prescriptions, and the esssay De Arthetica focuses on the study of an only illness, gout. The conceptual, methodological and linguistic differences between them constitute a good sample in order to analyse how the transmission of medical knowledge used to take place at the end of the Middle Ages. For Aldobrandino, experience is prior to
\end{abstract}

* Prof. Titular de Filología Italiana. UNED. Madrid.

1 Parte de este artículo ha sido ya publicado en italiano "Metodologia della trasmissione scientifica nel Medioevo", en Medicina nei secoli, Roma, Dipartimento di medicina Sperimentale e Patologia, Università «La Sapienza» (1999), vol. 11, n. ํ 1, pp. 43-55. 
Edad Media. Para Aldobrandino la experiencia se impone a la ciencia teórica adquirida en los libros, sin embargo Antonio Guainiero se coloca

en una posición equidistante entre saber científico y método empírico. La coexistencia de los dos tratados demuestra un estadio en el que se da una circulación paralela y una convivencia de diferentes sistemas de trabajo. the theoretical science acquired in books. However, Antonio Guainiero's position is equidistant between scientific knowledge and empirical method. The coexistence of both essays demonstrates the existence of a parallel and simultaneous circulation of different working systems.

En la biblioteca Nacional de Madrid y en la Biblioteca Capitular de Toledo están depositados dos manuscritos inéditos italianos relacionados ambos con la medicina, aunque cada uno de ellos desarrolla aspectos diferentes, bien sea desde el punto de vista argumental bien desde el punto de vista metodológico. El Spechio di medicina ${ }^{2}$ de Aldobrandino di Berto, que está en la Biblioteca Nacional, procede del convento de los Dominicos de Plasencia y es un tacuinum sanitatis en el que se recogen una serie de compuestos materializados a través de procedimientos y formas diferentes: aguas, aceites, vinos, distintos tipos de bebidas, electuarios, píldoras, polvos que, según su autor, poseen cualidades tan fuera de serie que son capaces de curar casi todas las enfermedades conocidas. El segundo texto, que se conserva en la Biblioteca Capitular de Toledo, procede del fondo Zelada y llegó allí en 1798, junto a otros manuscritos que el cardenal Zelada envió a su amigo el cardenal Lorenzana, a la sazón Primado de Toledo. Su título es De arthetica passione ${ }^{3}$, su autor el doctor Antonio Guainiero ${ }^{4}$ de Pauia y fue traducido al volgare (romance) por Antonio Cauchoreus.

Si el Spechio es un libro práctico que reúne una serie de recetas heterogéneas, desde la perspectiva médica, y la procedencia geográfica de cada una de ellas, el tratado De Arthetica, se concentra en el estudio de una única enfermedad en sus distintas manifestaciones. Se trata de un

2 Ms. n. 1458 de la Biblioteca Nacional de Madrid. Siglo xv, 62.ff. en 8. En el lomo: Spechio di medicina.

3 Ms. n. 98-6 de la Biblioteca Capitular de Toledo. Siglo xv, 62.ff. en folio. En el lomo de la encuadernación del XVIII Medic in volg. El texto original es del siglo XIV, pero la copia de Toledo procede de la mitad del XV.

4 Guainiero $\in S$ la forma registrada en el manuscrito. Los historiadores de la medicina que han estudiado su obra lo citan como Guainerio. 
estudio monográfico sobre la gota y las que, entonces, eran consideradas sus variedades: ciragra, podagra y ciática.

Las diferencias existentes entre ambos, que trataremos de ilustrar a continuación, constituyen un buen ejemplo para poder analizar cómo se lleva a cabo la transmisión de conocimientos médicos a finales de la Edad Media.

Atendiendo en primer lugar a la metodología empleada por los dos autores en la exposición de la materia médica se aprecian notables diferencias, como diferentes son también la manera de presentar la composición y distribución de los contenidos. De hecho, son precisamente las diferencias derivadas de la materia médica ilustrada en cada uno de los tratados las que condicionan la estructura y la modalidad de exposición de los conocimientos médicos.

El Spechio no toma como punto de partida ni el origen de la dolencia, ni el desarrollo de la afección, ni el tratamiento de la enfermedad que hay que curar, sino que se limita a enumerar una serie de remedios, paliativos y curativos que, según el autor, son tan poderosos y están tan firmemente experimentados, que tienen capacidad para curar enfermedades de origen y etiologia muy diversas ${ }^{5}$. Por el contrario, en el tratado De arthetica passione se percibe, desde la fase inicial, una intencionada voluntad de estructurar el conocimiento con rigor científico, por ello, el autor empieza dando primero la descripción de la enfermedad, explicando el origen, los síntomas y su interpretación, para terminar prescribiendo los remedios oportunos y necesarios en cada caso y los correspondientes procesos de elaboración.

Si el lugar de procedencia y la metodología son distintas, también lo es la tipología lingüística de ambos manuscritos. El Spechio di medicina nos sitúa lingüísticamente en el centro de Italia, en un área vernacular toscana, con marcas dialectales procedentes de la zona de Siena ${ }^{6}$, mientras que esta versión italiana del De Arthetica refleja ciertas características regionales que remiten a un tipo dialectal de cuño septentrional, localizado en las regiones de Lombardía, Trentino y Véneto ${ }^{7}$.

\footnotetext{
Prueba de ello es la virtud de l'aqua la quale si chiama madre dela natura que sana el «male di fiancho et del male de gotta asciaticha. Et del male ch[e] si chiama cholica passio, et del male del matrone. Et del male de la matre delle donne. Et al male che si chiama battiquore, et al male d'o[m]gni oppilatione di fegate...» $6 \mathrm{v}$.

6 Véase María Teresa NavarRo. «Aqua la quale si chiama madre de la natura: malattie e rimedi nello Specchio di medicina di Aldobrandino di Berto", en AA.VW. Atti del Terzo convegno della Società Internazionale de Lingüística e Filologia Italiana, Napoli, Edizioni Scientifiche Italiane, 1997, pp. 605-606.

7 Más concretamente a orillas del lago de Garda, como aparece atestado por ciertos têrminos ictiológicos: p[er]gie, carpionj, temalj, recomendados en el cap. XXVI, "Doue si mette el regimento del'arthetica pres[er]uatiuo. Et prima de le sei cose no[n] naturale».
} 
Otra diferencia radica en la motivación por la que ambos autores emprenden la redacción de sus escritos. No cabe duda de que el recetario de Aldobrandino di Berto tiene un móvil bien definido, el de ampliar lo máximo posible el radio de difusión de sus «milagrosas» recetas. Así el Spechio aparece constelado de autocitaciones y de reclamos que remiten a sus exclusivos preparados ${ }^{8}$. No obstante, en la redacción de este limitado vademecum se esconde otro móvil que tiene mucho que ver con la encendida polémica que durante la Edad Media opuso a los que enseñaban medicina teórica en el marco académico de las grandes universidades y a los «practicones» que ejercían su profesión, sin contacto con el mundo universitario, pero pagados de la experiencia adquirida en la práctica ${ }^{9}$ de la medicina, que les hacía enfrentarse diariamente a múltiples enfermedades y tratamientos.

Se puede aventurar la hipótesis de que Guainiero redacta su tratado sobre la gota como suma de las experiencias adquiridas como médico del balneario de Acqui, al que se acercaban ilustres personalidades de la vida política italiana que padecían esa enfermedad. Integrando práctica y teoría crea un tratado ordenado e inteligible, enriquecido con gran profusión de remedios, aptos para sanar diferentes tipos de gota, en especial la podagra o dolor del pie, la ciragra o dolor de la mano y la sciatica o dolor de la cadera, aunque no hace mención ni de la cloidagra, dolor de la clavícula ni de la gonagra, dolor de rodilla.

La claridad de exposición de la que hace gala, atraen al traductor, Antonio Cauchoreus, que decide emprender la versión italiana del De arthetica partiendo de una motivación más restringida y localizada. El punto de partida, tal y como afirma el mismo traductor, no es más que el de poder procurar alivio a la enfermedad contraída por el Magnifico señor Galeotto Malatesta ${ }^{10}$. Incluso siendo consciente de que su versión del De arthetica podría llegar a manos de gente "sencia disciplina medicinale" que al utilizarlo podrían «in errore cadere», aun así después de haber reflexionado que la «principale intenctione de l'auctore fu di giovare a quanti più si potesse» 60v emprende la traducción.

8 «(L)E nostre pillole chiamate angeliche son<n>0 pillole molte efficaci et di grandissima virtù. Et chiamamole nostre perché i[n] o[m]g[ni] nostra cosa l'adopariamo», 55v/ 56r.

- A propósito de esta polémica de la que trata el autor en una breve introducción filosóficomoral, que precede al Spechio, véase María Luisa ALTIERI BıAGI, Guglielmo volgare. Studio sul lessico della medicina medievale, Bologna, Forni, 1970, pp. 11-12.

10 Muerto en Cesena en 1385. Así se expresa en la epistola dedicatoria: «,..ha[m]mi mosso l'amore et la pietà mia v[er]so di te uedendoti in qualche modo da questa crudel passione menaciato", $60 \mathrm{v}$. 
Haciendo abstracción de las diferencias enumeradas, se da una coincidencia en la intención que vale la pena poner de manifiesto. Tanto el compilador del De Arhetica como el redactor del Spechio apuntan hacia la creación de una obra divulgativa, cuyo objetivo principal, no es únicamente el de transmitir una serie de conocimientos médicos, sino, por encima de todo, el de ampliar todo lo posible el campo de los potenciales usuarios. Sin embargo, la manera de actuar por lo que respecta a la exposición de la materia médica se desarrollará siguiendo caminos divergentes.

En realidad el autor del Spechio, Aldobrandino di Berto es lo que se podria definir como un «maistro manuale» y puede que, incluso sea un plagiario. Bien es cierto que en el periodo en el que realiza la compilación de su breve tratado sobre los remedios que curan determinadas enfermedades puede resultar atrevido hablar de plagio en sentido estricto, ya que, por entonces, la demanda de este tipo de manuales de enseñanza práctica estaba muy difundida, por lo que éstos se copiaban y se volvían a copiar, razón por la que gozaron de una amplísima divulgación. Por otra parte este tipo de procedimiento estaba reconocido por la tradición, ya que el mismo Mesué afirmaba que había escrito su tratado de farmacología porque algunos amigos le habían rogado que reuniera fórmulas médicas que hasta entonces se encontraban dispersas ${ }^{11}$. Lo mismo sucede con el Antidotario de Nicolò Preposito en el que el autor

comincia col dichiarare d'aver scritto il libro rogatus a quibusdam in practica medicina studere volentibus i quali desideravano conoscere il modo di preparare i diversi medicamenti e di sapere quanto di gomme, erbe, semi dovessero prendere per preparare un rimedio composto ${ }^{12}$.

Al estar, por entonces, la ciencia medica muy próxima a la escolástica y a la moral cristiana, la enseñanza teórica se realizaba en las universidades. Quien deseara aprender y ejercitar la medicina empírica y cotidiana tenía que seguir durante cierto tiempo a un médico que le enseñara la práctica y la forma de ejercer este tipo de medicina. Pero si en realidad Berto no fue un plagiario de contenidos si pudo serlo por lo que respecta al título completo de su obra: Spechio di medicina.[Opera]Composta et ordinata et scripta per me Aldobrandino di Berto delli Aldobrandini di Siena. Ahora bien, desde hacía tiempo otro Aldobrandino di Siena había com-

\footnotetext{
11 Véase Alberico BenedicentI, Malati, medici e farmacisti. Storia dei rimedi traverso i secoli e delle teorie che ne spiegano l'azione sullorganismo, Milano, Ulrico Hoepli, 1924, p. 354.

12 Ibid., p. 372.
} 
puesto un tratado médico, redactado en francés, el Régime du corps ${ }^{13}$ que, con la traducción de Zucchero Bencivenni, realizada en 1310, había alcanzado una extraordinaria circulación y, por la tanto un extensa notoridad. A pesar de la homonimia que se da entre los dos autores de Siena resulta bastante difícil establecer una relación de proximidad entre el Spechio y el Régime ${ }^{14}$, y cabe, más bien, la hipótesis de que el autor del cuaderno de recetas, sin tener en cuenta ahora cuál fuera su verdadero nombre, quisiera astutamente seguir la estela de éxito que había alcanzado la obra de su predecesor.

No es el caso del doctor Antonio Guainiero de Pavia, hombre de extensa formación científica que antes de compilar el De arthetica contaba ya en su haber con otra obra de tema médico el De febribus, que él mismo menciona en su tratado sobre la gota (59v), y también con un tratado sobre los baños De balneis, como recuerda en el último capítulo, dedicado a la terapia bainearia:

«No[n] uoglio piu oltra i[n] le lor laude procedere p[er] che quelle nel tractato de bagni altre uolte descripsi...

Por las noticias que Guainiero nos proporciona sobre su vida en el De arthetica se sabe que ejerció la medicina en Avignon y en Turín ${ }^{15}$. Hoy sabemos además que estudió en Pavía y en Padua, que ejerció la medicina en Venecia y en Lombardía, que posteriormente fue lector en Pavía (1412) y en Chieri (1428). Está considerado como uno de los mayores y mejores exponentes de la medicina del siglo xv y, para algunos, fue pionero en los estudios de anatomía patológica y experimentación en cadáveres de las alteraciones que provocan la muerte. Según refiere Pazzini ${ }^{16}$, la obra de Guainerio «consta de 16 volúmenes: nueve tratados sobre enfermedades de la cabeza, de los ojos, de las orejas, de la nariz, de la garganta, del pecho, de los pulmones, del corazón, del estómago y del útero; un tratado sobre la pleuritis (De pleuresi), un tratado sobre los efluvios morbosos del bajo vientre (De Fluxibus); un tratado sobre la artritis (De aegritudinibus

13 Véase Alfredo Schiaffini, Testi fiorentini del dugento e dei primi del Trecento, Firenze, G.C. Sansoni (1926), 1954, pp. 185-201; y también: Opera del Vocabolario Italiano. Bibliografia dei testi in volgare fino al 1375 preparati per lo spoglio lessicale, Firenze, Accademia della Crusca, 1992, pp. 439-440.

14 De hecho el Libro composto da Mo. Aldobrandino franzese et fatto vvigare da Mo Vghetto Benciven[n]i fiorenino, lanno MCCCX está estructurado en capítulos temáticos.

15 "...jo molte sciatiche ho curato et infra le altre vno jn Auignone et l'altro in Thaurino...», $41 \mathrm{v}$.

16 Véase Adalberto PAZZINI, Bozze di stampa, vol. 2. Biblioteca di Storia della Medicina, Università "La Sapienza», Roma, s.v. Guainerio. 
junctarum sive de arthetica); uno sobre los cálculos (De Calculosa passione), uno sobre la peste (De peste), un antidotario (Antidotarium) y uno sobre el balneario de Acqui (De Balneis Acquae civitatis antiquissimae commentariorus). Efectivamente, alrededor de 1435 Antonio Guainerio, médico de Ludovico de Acaja y de Amadeo VIII de Saboya era enviado a las termas de Acqui por Giangiacomo de Monferrato con la misión de verificar la verdadera eficacia de sus aguas termales ${ }^{17}$.

Es evidente que Guainiero posee una estensa formación teórica hermanada, por lo menos en el caso de las curas termales, con la experiencia práctica que ilustra con ejemplos concretos, como el del cardenal Giacomo degli Isolani «che i regolari bagni liberavano da una diffusa artritide onde n'erano impedite e l'andatura e l'equitazione». No se queda en los ejemplos sino que sistematiza los pasos a seguir, recomendando un conjunto de medidas encaminadas hacia una cura provechosa: beber agua mineral, la inmersión completa en las termas y los apósitos de barros calientes, fija en un periodo de quince días la duración media de los baños y establece el mes de mayo como el momento más propicio para beneficiarse de las aguas termales de Acqui ${ }^{18}$.

Por lo que respecta al tratado sobre la gota Guainerio demuestra sus conocimientos teóricos remitiendo con frecuencia al juicio de las autoridades, como en el caso de los Aforismos del Corpus Hippocraticum en el que se afirma que ni los eunucos, ni las mujeres fértiles ni los niños pueden padecer la gota ${ }^{19}$. Junto con Hipócrates son muchas las autoridades en las que se basa el médico italiano ${ }^{20}$. Los conocimientos teóricos de Guainerio están muy por encima de los del autor del Specchio, en el que la recurrencia a las autoridades es mínima ${ }^{21}$. Una vez establecida la diferencia entre ambos autores no parece extraño que el método empleado por cada uno de ellos en la transmisión de la ciencia médica esté en consonancia con su propio nivel de sabiduría.

Analizando, pues, el método utilizado por nuestros dos autores en la descripción y curación de la gota se perfilan dos procedimientos bastante

\footnotetext{
17 Véase Lorenzo GuALDINo, "Le Terme acquesi all'epoca del Rinascimento italiano", en Minerva Medica, XXIII (1932), vol. I, n. ${ }^{\circ} 23$, p. 802.

18 Ibid., pp. 802-4.

«...et il nostro serenissimo Ypocrate ( ). Eunuchi no[n] podagrigant e[t] c[aetera]», $1 \mathrm{r}$.

20 Son frecuentes las citas de Galeno, Filagrio, Andrómaco, Demócrito, Ciranides, Apuleyo, Cornelio Celso y Sesto Plácido; arabes: Avicena, Averroes, Avenzoar, Mesue, Rasis y Serapión el Joven; italianas: Gentile da Foligno, Mondino de' Liucci y Nicolao da Reggio.

${ }_{21}$ Cita soltanto Aristoteles y Avicena, a un Salmista? y el Libro del cielo e mundo de San Agustín.
} 
diferenciados. Aldobrandino procede en su recetario señalando, de manera dispersa y desordenada, algunos remedios compuestos que benefician no sólo al que padece de gota, sino también a los enfermos que han contraido muchas otras dolencias. Por lo que respecta a la estructura, la disposición es bastante sencilla: da en primer lugar el nombre del remedio compuesto, explica inmediatamente las virtudes que la experiencia ha venido asignando a cada uno de los compuestos citados y, en ciertos casos incluye ejemplos escogidos para reforzar el valor terapeútico de la receta ${ }^{22}$. Para demostrar la fuerza de actuación del compuesto enuncia un número bastante elevado de enfermedades susceptibles de ser curadas por el preparado en cuestión. Luego da una lista de todos los simples que integran la composición magistral y señala las dosis y el proceso de elaboración.

El doctor de la corte de Saboya actúa más metódicamente, procede con orden y coherencia como demuestra la propia estructura del tratado sobre la gota. El De arthetica está dividido en treinta capítulos y, a pesar de que el ms. de la Capitular de Toledo es acéfalo (falta el primer capítulo), podemos saber por el índice, incluido al final de la obra, que su título rezaba: De la notificazione de l'arthetica et de alcune sue specie. Cabe suponer, por el rigor expositivo del que hace gala en los siguientes capítulos, que el capítulo perdido estuviera dedicado a la exposición teórica de la enfermedad y, quiza, a las definiciones de los distintos tipos de gota.

En los capítulos siguientes proporciona una descripción genérica de la gota, con referencias a lo específico de cada variedad: la ciragra y la podagra, para después enumerar distintos remedios útiles para sanar los dos tipos de afección. Después describe qué es la ciática o gota de la cadera y dedica los últimos capítulos a la medicina preventiva. Así, pues, en los cuatro primeros capítulos se propone una exposición genérica de las manifestaciones de la gota, etiología, síntomas y fármacos adecuados de aplicación común. Entre el quinto y el undécimo se define la ciragra, síntomas, remedios, procesos de digestión y evacuación. En el capítulo duodécimo se estudia la podagra, síntomas, remedios y formas de digerir y evacuar la materia. En los capítulos comprendidos entre el decimotercero y el decimonoveno se proponen remedios aplicables a las diferentes clases de gota, que calman el sufrimiento e impiden que el fluir de los humores cristalice en las articulaciones. Sigue después la descripción de ciertos remedios, narcóticos y no narcóticos, que disuelven la materia causante de

22. Come accade con le Pillole chiamate imperiali, «...gieneralme[n]te p[re]seruano la ca[r]ne da omg[ni] corruptione. Impero ch[é] le cose ch[e] ci entrano sonno satisfate ch[e] tene[n]done in vno co[r]po mo[r]to, no[n] si corromparebbe mai», $54 \mathrm{v}$. 
la dolencia. Entre el vigésimo y el vigésimo cuarto capítulo se trata de la ciática, síntomas, y remedios para debilitar el dolor y curar el mal. El capítulo siguiente está dedicado al régimen que debe seguir el enfermo de ciática, especialmente en los momentos de paroxismo. Los últimos capítulos tienen por objeto la medicina preventiva: dieta, sangrías y otras variedades de purgantes y, para finalizar, el autor se extiende en la descripción de ciertos experimentos que sirven para paliar el dolor e impedir el paroxismo, recalcando que son métodos tomados de los antiguos y utilizados por las autoridades ${ }^{23}$. Tratándose de un experto en terapia balnear, como ya se ha visto, el tratado termina con una capítulo en el que se recomiendan los baños, naturales o artificiales, por su bondad y utilidad.

Pongamos ahora frente a frente las propuestas que sugieren Aldobrandino di Berto y Antonio Guainiero para curar la enfermedad de la gota.

Para curar cualquiera de los tipos de gota, bien sea ciragra, podagra o ciática el autor del Spechio aconseja el uso de cinco compuestos:
Aqua la quale si chiama madre dela/natura $5 r$, olio chiamato Imperiale $29 \mathrm{v}$,
olio da gotti, el quale fu composto da Aristotile,40r/v, pillole chiamate imperiali $54 \mathrm{r}$ pilloli (sic) cniamate angeliche $56 \mathrm{v}$

Las pildoras Imperiales ejercen funciones preventivas puesto que «preseruano gli artetici et li podagri», 54r. El aceite de gota es paliativo y quita "omg[ni] spasimo et doglia di gotta", 40v. Las pildoras angélicas son curativas porque «nettano gli omori nociui», $56 \mathrm{v}$ y también lo es el aceite imperial ya que «se l'ognierai d'ogni tre o quatro hore co[n]sumarà tucto l'omore", 33v. Pero entre todos los compuestos recomendados en el cuaderno de Aldobrandino el más perfecto de todos es el agua madre de la naturaleza porque consigue la curasión de la gota.

«Et similme[n]te q[ue]sta aqua è bonissima [...] et colui che la usarà come è detto $\mathrm{i}[\mathrm{n}]$ brevissimo tempo ne sarà liberato. Et etia[m] sarà liberato d'omg[n]i male di fiancho et del male de gotta asciaticha...s, $6 \mathrm{r} / 6 \mathrm{v}$.

Ahora bien aquí se pone de manifiesto una de las muchas contradicciones que se pueden captar en el Spechio, porque, si el agua madre de la naturaleza en sí misma tiene poder como para sanar la ciática, si se usa

23 «Ancora, il sterco di lupo in pelle di animale da lui morto sopra se portato da l'arthetica preserua, como dice Apuleo», 55r. 
en combinación con el aceite imperial solo consigue paliar el dolor (f. 33v/34r). $Y$, además, cada uno de los compuestos citados sirve, no sólo para curar la gota, sino que se recomienda para el tratamiento de múltiples enfermedades.

En el De arthetica, por el contrario, antes de llegar a proponer cualquier remedio para curar la gota se incluye una reflexión teórica que trata de explicar las causas, es decir cuáles son los humores que generan la enfermedad, la interpretación correcta de los síntomas, a lo que sigue la prescripción del compuesto más adecuado al tipo de afección. Para enseñar a formular un diagnóstico correcto Guainiero se sirve de la pareja color-sintoma, a través de la cual enseña a reconocer cuál es la procedencia de la afección y como curarla.

Observando el color del «paciente membro» se puede aprender si el origen de la gota procede de flema, cólera, sangre o melancolía. Es necesario, por lo tanto, saber interpretar los «signos demostrativos» de la enfermedad. Si el origen de la dolencia procede del humor sanguineo el miembro enfermo tiende a enrojecer: "se quello sangue sia [...] il colore del me [m]bro ad roseccia tende...", $4 \mathrm{v}$. Si, por el contrario el origen es de humor colérico el color del miembro tiene a amarillear: «La collera fa il dolore pungitiuo et molto intenso et se 'I membro caldo sia ad alcuna citrinità tendendo cu[m] niuno o poco tumore", $4 \mathrm{v}$. En el caso de que proceda de humor flemático, el color del miembro tratado varía muy poco con respecto al que está sano: «ll fleg[mati]co humore no[n] causa molto intenso dolore [...] el colore suo dal sano molto no[n] è distante», $5 \mathrm{r}$. Y si su origen está ligado al humor melancólico el color puede tender hacia el negro: «El membro liuido o al negro declina et le più fiate toccando freddo appare» $5 r^{24}$.

Para el adoctrinamiento el médico de corte utiliza un método progresivo, en el que la introducción de los contenidos aparece sabiamente dosificada. Una vez que el destinatario de su tratado está ya en disposición de diagnosticar, según el color del miembro enfermo, originado por la disfunción de un solo humor, pasa a explicar que la dolencia puede estar causada por humores «permisti», por lo que, si en ese caso se diagnosticara sólo en base al color del miembro se podría errar fácilmente.

"Ma sapie che in tali humorij misti il colore et calidità del membro facilmente poteriano i[n]ga[n]nare $\mathrm{p}[\mathrm{er}]$ che la collera [con] flema $\mathrm{p}$ [er]mista alla parte di sopra del membro facilmente discorre, p[er] la qual cosa il membro

24 Las cursivas son mías. 
allora i[n]rosisse e tende al citrino et anche se riscalda ben che dentro de la giuntura sia fiema che faccia dolore, de che appare se alhora q[ue]sto da collera prouenire tu judicasti in $n$ [on] picolo ma grande er(r)ore caderesti», $5 \mathrm{v}$.

El maestro de la Arthetica enseña metódicamente que la base de una medicina eficaz es un diagnóstico correcto, sin el cual resultaría imposible aplicar los remedios pertinentes y el doctor Guainiero utiliza para curar la gota el ermodattilo, el hermodátil o cólchico efemerón, según DioscóridesLaguna ${ }^{25}$ que, a partir del siglo vII, se convertirá en un clásico en el tratamiento de la gota ${ }^{26}$. Benedicenti cita al malagueño Ibn-Beitar y su obra Libro de los simples en la que se utiliza por vez primera el cólchico otoñal para curar la gota ${ }^{27}$. Sin embargo el autor del Spechio no utiliza en ningún caso este simple, ni siquiera en el maravillosos compuesto que el llama Aqua la quale si chiama madre de la natura que contiene más de cincuenta elementos ${ }^{28}$.

Por la que hasta aquí se ha ido exponiendo, de la comparación de los dos manuscritos médicos se pueden extraer algunas conclusiones, útiles para iluminar algunos aspectos del ejercicio de la profesión médica en un momento cronológico determinado, situado a mediados del siglo XV en Italia.

En primer lugar cabe destacar al uso de la terminología médica utilizada por Berto y por Guainiero (o por su traductor Cauchoreus). El Spechio, siendo como es menos riguroso en otros aspectos de la transmisión, para referirse a la enfermedad se sirve de un muestrario más amplio de términos. Utiliza por una parte el vocablo de origen latino: gotta, (gotte/gotti artetiche), de formación más reciente y de carácter más popular ${ }^{29}$, junto a formas de origen griego: (ciragra, podagra/potagra). El De arthetica, por

25 María Teresa Herrera, Diccionario esfañol de términos médicos antiguos, Madrid, Arco Libros, s.v. hermodátil.

26 Angelo Ceconi, La gotta, Torino, Edizioni Minerva Medica, 1929, pp. 8-9.

27 Alberico BenEdicenTl, op. cit., p. 394.

28 Perle da pestare, Moscato finissimo, Ambra di balena, Oro fino i[n] panelle, Choralli rossi, Choralli bianchi, Ciennamomo electo, Garofani scielti, Noci moscade, Spigo nardo, Mace belle, Granasole, Anasi crudi, Chubebe, Grana paradisi, 8v Pepe longo, Pepe Giengiono, Legno aloe, Galanga, Çettuaria,Regolitio, Sticatos, Çaffarano, Baccare, Cardamomo, Calamo aromatico,Seme di basilico, Seme d'a[r]temisia, Gientiana, Sassifragia, Dittamo, Torme[n]tilla, 9r Asse[n]tio, Saluia domestica, Ruta, Jsopo, Menta, Maiorana, Camedreos, Camepiteos, Calame[n]to, Origano, Santuregia, Celidonia, Cientuaur[e]a, Bretonica, Eufrasia, Jua, Fiore di sanbuco, Fiore di ramerino, 9v Rose rosse, Rose bianche, Mando[r]le dolci, Pignioli, Seme d'edera, Seme di gin[e]pa[r]o, Vua passola, Orbachelle, Scorçe di cedro, Sco[r]çe d'aranci, Fichi sechi, Dattari, Mele bello, Çucharo di tre cotte, Aqua uite fina.

${ }_{29}$ Véase Adalberto PAZzINI, «Cenni storici sulla eziologia e la patogenesi della gotta», en Scritti di storia delle malattie, Roma, 1968, p. 35. 
tratarse de un estudio más profundo y riguroso, sigue la tradición de los textos antiguos, prefiere la dicción griega y evita el uso de la forma latina ${ }^{30}$.

Por otra parte, se da un contraste en la valoración que hacen ambos entre medicina teórica y medicina práctica. Aldobrandino no concede ningún valor a la medicina teórica, incluso se permite afirmar:

"oggi dì se n'è fata cie[r]ta pratica et no[n] s'os[er]ua lo stile et il modo degli antiqui», $3 \mathrm{v}$.

Por lo tanto la experiencia se impone a la ciencia teórica adquirida en los libros. Esta afirmación presupone, quizá, una visión más moderna de enfocar la relación entre teoría y práctica, puesto que se encamina hacia la ruptura de todo enlace con las autoridades, favorecido y reforzado por la tradición, y se propone dar mayor peso al valor de la experiencia en la curación de enfermedades. No es por casualidad -dice él一, por lo que ante los ojos de Dios las dos son comparables:

«...Dio a co[n]ciessa questa scie[n]tia della /medicina vniuersalme[n]te a tucti li homini [...] Alcuni l'a conciessa per reuelatio/ne. Alcuni p[er] scie[n]tia et a alcuni altri per experie[n]tia», 3v.

Antonio Guainiero se coloca, sin embargo, en una posoción equidistante entre saber científico y método empírico y lo dice expresamente en la epístola dedicatoria a Malatesta, donde menciona la necesidad de poseer la "disciplina medicinale» con el fin de poder manipular los saberes sin correr el riesgo de hacer daño al enfermo. En su tratado, cuando se refiere a los humores que pueden generar la podagra, ejemplifica brevemente las contradicciones que se pueden captar en los juicios que Galeno, Avicena y Rasis, han expuesto sobre ese problema, pero considera que la discusión no aporta ningun beneficio al enfermo y, por lo tanto, no deben ser tenidas en cuenta:

«Et cussi appare quo[n]da[m] modo affirmare il contrario di quello ch[e] di sopra dicto habiamo. Et p[er] che il dechiarare in questo loco tale disceptation[e] seria a noi poco utile, al proposito nostro adonca venendo...", 3v.

Quien decide enseñar medicina está obligado a conocer la teoría, pero Guainiero considera que no hay que confundir al paciente con palabras que ni

30 Para el problema terminológico sería de gran utilidad poder confrontar la traducción con el original latino. 
siquiera puede entender y de poco o de nada le sirven en el tratamiento de su enfermedad. El De Arthetica se presenta, de hecho, como un tratado equilibrado entre empirismo y ciencia teórica y no sólo eso, porque a la vez que se mantiene en un nivel técnico más que aceptable, consigue acercar a personas no cualificadas el mundo de la enfermedad, con sus síntomas, procesos y remedios. Para ello se apoya en ciertos procedimientos didácticos extraordinariamente originales, ya que introduce una variante en el modo de acercar la enfermedad al enfermo que no he encontrado en otros manuscritos del mismo género. Con el fín de establecer una cierta familiaridad entre dolencia y enfermo recurre a una figura retórica propia de la fábula: la personificación de la enfermedad. La afección se convierte así en una noble dama:

«La nobile Mado[n]na Arthetica tutte le regione jn ciascuno anno visita, et li habita[n]ti di quelle piu potenti, la quale è a loro tanto amoreuole che se alchuno di loro p[er] la mano, per la scya o p[er] le pede piglia, e come spesse uolte accade, sego p[er] vno mese o piu oltra dimori, quasi fin ala morte no[n] l'abandona. Et adcioché cum luj piu longamente dimorare si possa, tuti li soi primi assalti quasi a ogni homo jncogniti li fa, p[er] che come dice A. et anche li antichi phisici p[er] exp[er]iencia imparorono se alhora q[ue]lla cognosciuta fosse, facilmente no[n] apta a ritrouare maj piu si descaciar[i]a...», 3v.

De este tratado sobre la gota se desprende una clara vocación científica y didáctica que preludia formas nuevas de sistematización presentes en otros tratados médicos de mediados del XV como il Librecto di Pestilen$\mathrm{Cia}^{31}$ di Nicolò d'Ingegne (1448) ${ }^{32}$.

La comparación establecida entre estos dos manuscritos pone de manifiesto que tanto el autor del Spechio di medicina como el de De arthetica passione ejercieron la práctica de la medicina, pero de manera diferente. El primero mostrando una actitud beligerante hacia la ciencia teórica, a la que mide con el mismo rasero que el de la medicina práctica. El segundo tratando de buscar una vía intermedia en la que poder integrar conocimiento teórico y casuística. Uno se limita a suministrar una lista de remedios, el otro, aunque unido a la fase de la medicina técnica que cree todavía en los remedios mágicos ${ }^{33}$, va más allá, propone que la ciencia

${ }^{31}$ En el que se definen científicamente términos que hacen referencia a enfermedades que, a menudo, se confunden con la peste: «Avemo nellj librj di medichina queste diuersità de nomj: peste, pestilencia, epjdimia, vndimia, mortalità et febre pestilen/ciata [...] secundo el mio judicio grandemente differ[e]nti son $<n>0 . .3 v 1 / 3 v 2$.

32 Sobre el Librecto véase Pietro Sısтo Due medici, il principe di Taranto e la Peste, Napoli, Istituto Nazionale di Studi sul Rinascimento Meridionale, Napoli, 1986.

${ }^{33}$ «Ancora, né sole né luna lucente se tu tagliarai il pe destro de la rana et in pelle di ceruo ligato sopra il pe destro de lo infermo per tri dí si portarà ligato[...] sedarà il dolore et farassi in perpetuo da podagra securo", $54 \mathrm{v}$. 
teórica tiene que ir de la mano del método empírico y, puesto que el objetivo principal es el de llegar al número más grande posible de destinatarios, escoge la estructura y el lenguaje adecuados, dando muestras de una notable capacidad pedagógica.

En realidad ambos son compiladores, aunque uno se basa en la medicina popular y el otro sigue a las autoridades, porque en aquella época quienes se dedicaban a la ciencia erano meno preoccupati di inventare che di raccogliere e che erano più sinceramente desiderosi di sapere che di creare qualche cosa di nuovo o d'originale ${ }^{34}$.

En cualquier caso la impostación tan distinta que proponen las dos obras son prueba de que hubo un periodo a finales de la Edad Media en el que se dieron simultáneamente tanto una circulación paralela, como una convivencia de sistemas de trabajo, que coexistieron durante una época. De los dos manuscritos inéditos de los que hemos tratado, el De arthetica passione, a pesar de sus fluctuaciones e inseguridades, cumple una breve etapa del largo camino que desembocará posteriormente en la medicina científica moderna. 